\title{
MICROSCOPY TECHNIQUES USED TO ELUCIDATE SURVIVAL STRATEGIES OF AN INTRACELLULAR PATHOGEN
}

\author{
Elizabeth R. Fischer*, Bryan T. Hansen**, and Ted Hackstadt*
}

*NIH/NIAID/Rocky Mountain Labs, 903 South 4 Street, Hamilton, MT 59840, **Western Washington University, 510 Nash Hall, Bellingham, WA 98225.

Developments in preparative microscopy tools and techniques combined with modern biochemical and molecular methods have improved our ability to relate structure to function and may facilitate our understanding survival strategies used by intracellular parasites. Survival by intracellular pathogens requires adaptation or modification of the host environment to avoid lysosomal fusion. Here, we demonstrate tools and techniques important for characterizing survival mechanisms of chlamydiae, an obligate intracellular parasite infecting epithelial cells to cause disease.

Conventional studies by TEM provide useful information about morphologic changes during the chlamydial developmental cycle. The infective metabolically inert elementary body (EB) has a distinctive condensed nucleoid (Fig.1a). Upon entry into host cells, EBs reside in a membranous compartment termed an inclusion, and after a few hours the nucleoid decondenses and the cells differentiate into the metabolically active reticulate body (RB) (Fig.1b). As RBs divide by binary fission, the inclusion membranes merge into one large parasitophorous vacuole, and after 18 hours (Fig. 1c) begin to differentiate back into EBs, before release 40-72 hours after the initial infection (Fig. 1d).

Antibody labeling of endosomal markers followed by horseradish peroxidase secondary conjugates and subsequent incubation with DAB substrate yields a highly specific electron dense reaction product demonstrating an early departure of chlamydia from the endocytic pathway during infection (Fig. 2a-c ). [1] Ceramide trafficking studies reveal interception of an exocytic pathway by the bacterial vacuole to create an intracellular niche for survival and growth (Fig. 2d ). [2]

Conventional SEM has demonstrated evidence of cytoskeletal rearrangement during attachment of EBs to the surface of host cells (Fig. 3a,b,\& d), and labeling with GFP-actin demonstrates actin recruitment during this process (Fig. 3c).[3] Actin remodeling can be induced by bacterial effector proteins secreted by a type III secretion system (T3SS). By immunoperoxidase staining (Fig. 3e), we localized a tyrosinephosphorylated chlamydial protein at the EB attachment site on HeLa cells. This event occurs in conjunction with actin rearrangements, possibly representing a signal triggering host cell responses. Detergent extraction of host cells allows visualization of colloidal gold-labeled EBs attached to the phalloidin stabilized cytoskeleton (Fig. 3f).

High pressure freezing and fracturing for cryo-SEM preparation revealed intracellular spatial information of host/parasite interactions with improved structural integrity (Fig. 4a). Extraction of cytoplasmic components by fixation with osmium and tannic acid prior to plunge freezing, exposed structures for improved depth of visualization within the inclusion (Fig. 4b). 


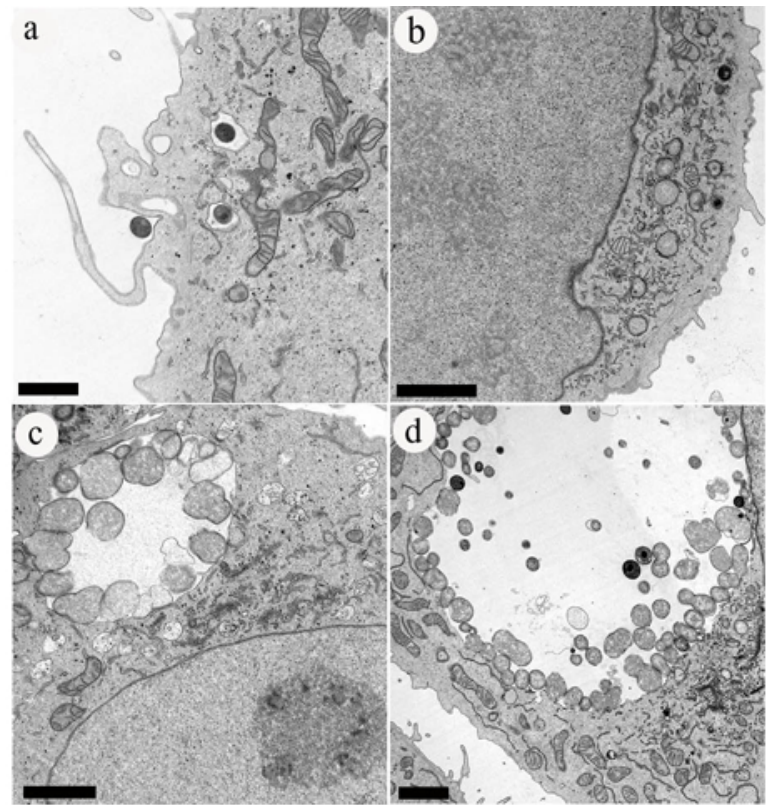

Fig. 1 Developmental cycle of Chlamydia trachomatis in HeLa cells at 0 h p.i. (a), 4 h p.i. (b), $18 \mathrm{~h}$ p.i. (c), and $24 \mathrm{~h}$ p.i. (d). Scale bars $=1$ micron

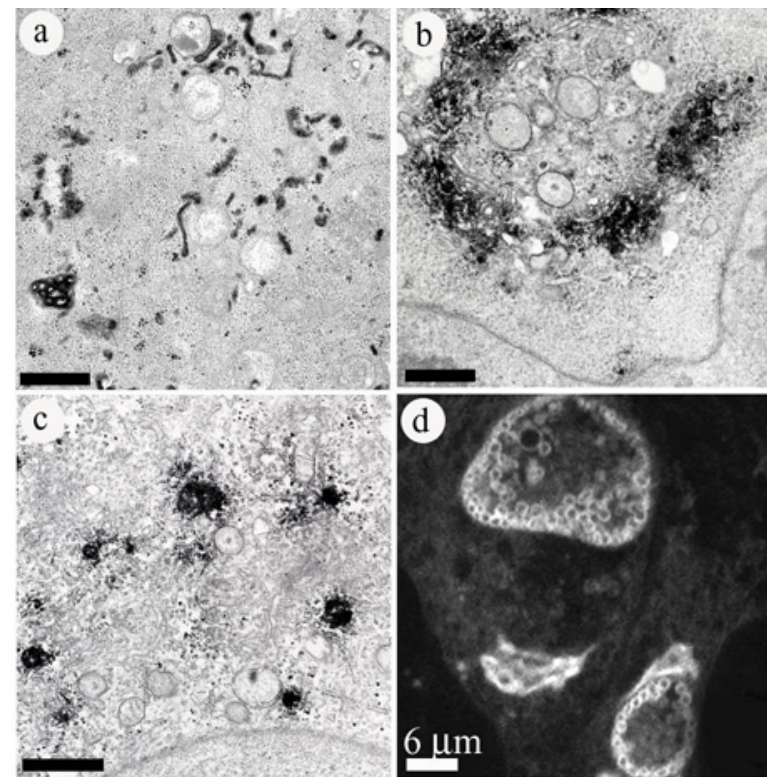

Fig. 2 Labeling with endosomal/lysomal markers at $4 \mathrm{~h}$. p.i. including transferrin (a), M6PR (b), LAMP 1 (c) shows association but not incorporation into the inclusion membrane. A fluorescent ceramide analog shows incorporation of Golgi derived lipids with the inclusion membrane and chlamydiae (d). Scale bars $=0.5$ microns

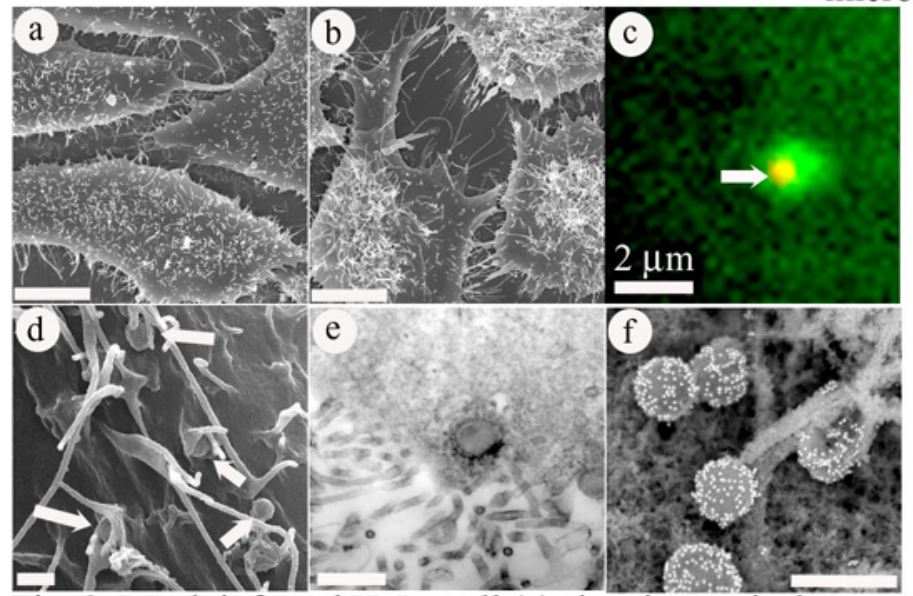

Fig. 3 Mock infected HeLa cell (a) showing typical structure compared to a chlamydia-infected cell $10 \mathrm{~min}$ p.i. exhibiting microvillar hypertrophy (b) and pedestal-like formation of the host cell in response to chlamydial attachment (arrows) (d). GFP- actin demonstrates the high concentration of actin in proximity to an attached EB (arrow) (c). Scale bars a-c $=5$ microns, scale bars $\mathrm{d}-\mathrm{f}=0.5$ microns.

\section{References:}

1. Scidmore, M.A., et al, Infect. \& Immun. (2003)71:973-984

2. Hackstadt, T. et al, EMBO J. (1996)15:964-977

3. Carabeo, R.A., et al, Infect. \& Immun. (2002)70:3793-3803

4. Clifton, D.R., PNAS (2004)101:10166-10171

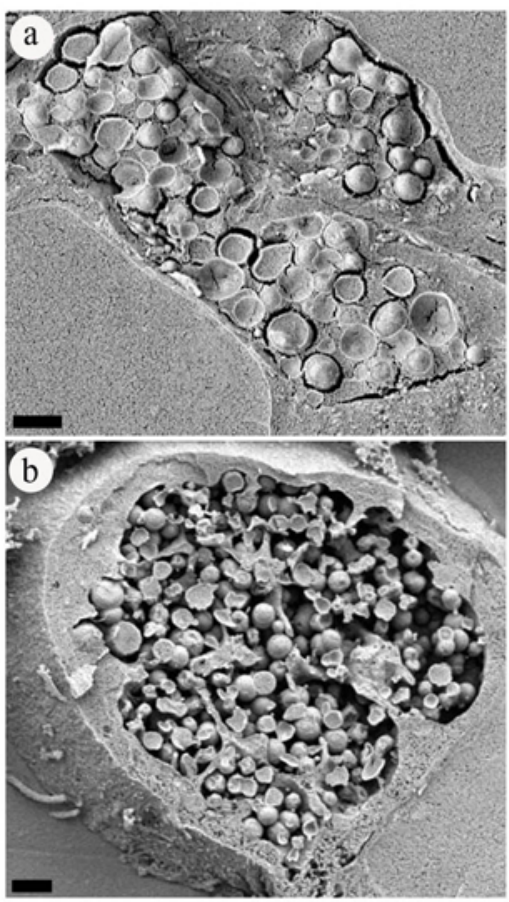

Fig. 4 High pressure frozen (a) compared to fixed plunge frozen (b) chlamydial inclusion. Scale bar $=1$ micron. 